\title{
Routing, Modulation, Spectrum and Launch Power Assignment to Maximize the Traffic Throughput of a Nonlinear Optical Mesh Network
}

\author{
David J. Ives · Polina Bayvel · Seb J. Savory
}

Received: date / Accepted: date

\begin{abstract}
We investigate the optimization of routing, modulation format adaptation, spectral and launch power assignment as a means of improving the utilization of limited network resources and increasing the network throughput. We consider a transparent optical network operating in the nonlinear transmission regime and using the latest software adapted coherent optical techniques. We separate the problem into one of routing, modulation adaption and channel assignment, followed by channel spectral assignment, and launch power allocation. It is shown, for three test networks, that the launch power allocation and channel spectral assignment can improve the transmission SNR margin over the fixed modulation, fixed power, fully load link worst case by approximately 3-4 dB. This increase in SNR margin can be utilized through modulation format adaption to increase the overall network throughput. This paper highlights that increased gains in network throughput can be achieved in nonlinear impaired networks when individual transmitter spectral assignment and launch power is optimized to minimize the nonlinear interference.
\end{abstract}

This work was supported by the UK Engineering and Physical Sciences Research Council, through the Centre for Doctoral Training in Photonics Systems Development, EP/G037256/1 and programme grant UNLOC, EP/J017582/1, and The Royal Academy of Engineering / The Leverhulme Trust.

\section{Ives}

Department of Electronic and Electrical Engineering,

University College London, London, UK.

E-mail: d.ives@ee.ucl.ac.uk

P. Bayvel

Department of Electronic and Electrical Engineering,

University College London, London, UK.

E-mail: p.bayvel@ucl.ac.uk

S. Savory

Department of Electronic and Electrical Engineering,

University College London, London, UK.

E-mail:s.savory@ucl.ac.uk
Keywords Off-line Routing · Nonlinear Impairments · Modulation Adaption · Power Optimization

\section{Introduction}

Optical fibre mesh networks connecting re-configurable optical add drop multiplexers, ROADMs, form the backbone of modern communications and the internet. These networks utilize wavelength routing to transparently connect source and destination nodes. Traditionally the transmitter-receiver pairs are designed to operate error-free in the worst case, for transmission between the furthest spaced nodes so that any route reconfiguration can be accommodated. This leads to over provisioning of resource with large margins for the majority of signals. With the development of software-defined transceivers [1-3] the transmitter can be re-configured allowing the transmission parameters to be adapted to the selected physical route. Given the continuing increases in network traffic and this ability to configure the transmitter parameters the physical network resources of bandwidth and power can be used more efficiently.

In this paper we consider a fixed network infrastructure with a physical topology of nodes, consisting of ROADMs and transceivers, connected by edges formed of dispersion uncompensated fibre pairs. Each fibre edge is formed in fixed spans with Erbium doped fibre amplifiers compensating for the loss of each span. Polarization multiplexed-mQAM modulated signals are transmitted on a fixed DWDM grid and routed transparently without wavelength conversion. A nonlinear quality of transmission model estimates the SNR of each signal while taking the individual launch powers into account. We use the model to optimizing the routing, modulation format, spectral assignment and launch power with the primary aim of maximizing the network data throughput. We extend our earlier work[4] 
by assessing the applicability of the optimization technique to more network topologies with different scale.

In the area of optical network optimization mixed line rates utilizing OOK, DQPSK and PM-QPSK signals with impairment-aware routing to reduce capital expenditure have been used[5], although this type of analysis makes it difficult to link cost savings with potential increases in traffic throughput. The recent development of elastic optical networks $[6,7]$ has improved spectral efficiency, however in this paper we only consider a fixed DWDM grid, leaving the option of utilizing the potential gain of "elasticity" in future work. The extension of elastic optical networks to include modulation format adaptation based on the quality of transmission or reach has also further improved overall spectral efficiency[8-10]. In this paper we use modulation format adaptation, based on the physical link properties, specifically, the nonlinear quality of transmission.

In traditional dispersion managed OOK systems, FWM is the dominant nonlinear impairment. It has been shown that for a sparsely populated spectrum by careful allocation of the channel centre frequency away from a regular grid the FWM components can be generated away from the transmission signals eliminating the interference at the expense of an increase in bandwidth usage[11]. Adhya et al [12] considered the FWM in the design of a logical network on the physical layer and equalised the SNR across channels by routing longer paths to the outer parts of the spectrum to reduce the number of interfering FWM components. In this work we consider the new high dispersion paradigm such that FWM is considerably reduced. We consider a situation where the spectral bandwidth is heavily occupied and the use of coherent transmission formats lead to nonlinear interference that is dominated by SPM and XPM

Other authors have included nonlinear impairments in the routing and spectral assignment algorithms by including a nonlinearity constraint[13] and through a power optimized reach constraint[14]. Here, we wish to take advantage of the results presented in[15] which indicate that the correct routing and spectral assignment can lead to improved SNR and throughput. We include a nonlinear weight in the optimization objective function to initially group highly interfering channels so that highly interfering channels can be subsequently given well separated spectral assignments. Finally individual transmitter launch powers were optimized, to maximize the reach[14] while in [16] an altruistic minimization of transmitter power was found to benefit the wider network. Here we take a global view of the network and adjust individual transmitter powers to equally distribute and maximize the available SNR margin above error free transmission.

In Sect. 2, we describe the nonlinear quality of transmission model used to assess the physical layer performance and allow adjustment of the individual transmitter launch powers. In Sect. 3 we describe the network physical layer parameters and test topologies used. This is followed by a description of the routing, modulation and channel assignment algorithm that is used to maximise the network data throuhput based on an ILP formulation in Sect. 4.1. The spectral assignment of the channels and the adjustment of individual transmitter launch power to maximise the SNR margin is described in Sect. 4.2. The final sections describe the application of the algorithms to a selection of real network topologies and demonstrate the gains in network data throughput that can be achieved.

\section{Physical Layer Nonlinear Propagation Model}

For the optimization considered in this paper a simple physical layer impairment model is required to allow rapid computation of the quality of signal transmission. The assumed signals formats are restricted to polarization-multiplexed coherent modulation schemes such that the linear impairments can be ideally and adaptively compensated in the coherent receiver. Signal degradation is caused by the combination of the accumulated ASE noise from the erbium doped fibre amplifiers, EDFAs, in the optical path and the accumulated nonlinear interference between co-propagating signals. For optical fibre infrastructure, that is dispersion uncompensated the Gaussian noise, GN, model has been assumed for the purpose of predicting and quantifying the nonlinear interference as a source of additive Gaussian noise[17-20] ${ }^{1}$. In this case the symbol SNR, $S N R_{i}$, of the $i^{\text {th }}$ signal is given by

$S N R_{i}=\frac{p_{i}}{n_{A S E, i}+n_{N L I, i}}$

where $p_{i}$ is the received signal power, $n_{A S E, i}$ is the ASE noise power and $n_{N L I, i}$ is the nonlinear interference noise power within the receiver filter bandwidth, all on the $i^{\text {th }}$ signal.

As the transmission loss of each span is fully compensated by the EDFA gain, the received signal power is equal to the transmitter launch power and the ASE noise power is given by $[24,25]$

$n_{A S E, i}=N_{S} 10^{\frac{N F}{10}} h v R 10^{\frac{A_{\text {span }}}{10}}$

where $N F$ is the amplifier noise figure $(\mathrm{dB}), h$ is Planck's constant $\left(6.626 \times 10^{-34} \mathrm{~J} . \mathrm{s}\right), v$ is the channel carrier optical frequency $\left(193.5 \times 10^{12} \mathrm{~Hz}\right), R$ is the symbol rate (Baud),

\footnotetext{
1 Recently a number of authors[21-23] have proposed correction terms to the GN model to overcome some of the GN models shortcomings particularly in the early spans of a transmission link where the accumulated chromatic dispersion is low. These correction terms reduce the expected nonlinear interference noise power for the modulation formats considered in this paper. We thus utilize the simpler GN model here under the assumption that it conservatively estimates the nonlinear interference noise and thus allows for robust network optimization.
} 
$A_{\text {span }}$ is the loss of a single fibre span (dB) and $N_{s}$ is the number of spans in the route. This approximation for the ASE noise assumes that the gain of the amplifier is large and that any reduction in gain needed to match the span loss is achieved using a following variable attenuator. The effective noise bandwidth used is equal to the symbol rate $R$ under the assumption that the overall noise spectrum is white, dominated by ASE, and the receiver implements an ideal matched filter to maximize the SNR.

The Kerr effect leads to nonlinear interference noise that can be considered as self phase modulation, SPM, for nonlinear interference caused solely within the $i^{\text {th }}$ signal, cross phase modulation, XPM, for interference on the $i^{\text {th }}$ signal caused by the $j^{\text {th }}$ signal and four wave mixing, FWM, for interference caused by the interaction of multiple signals. For well spaced signals, such that there is negligible crosstalk of the nonlinear interference noise between signals, and under the assumption that the nonlinear interference noise due to FWM can be ignored as insignificant ${ }^{2}$ [18] then the nonlinear interference noise, $n_{N L I, i}$ on the $i^{\text {th }}$ signal due to SPM and XPM can be written as [15,26,27]

$n_{N L I, i}=N_{s} p_{i} \sum_{j} X_{i, j} p_{j}^{2}$

where $N_{s}$ is the number of spans over which the nonlinear interference accumulates, $\mathbf{p}$ are the signal launch powers and the summation $j$ is over all interacting signals. $X_{i, j}$ is a single-span efficiency factor for the nonlinear interference noise on the $i^{\text {th }}$ signal caused by the $j^{\text {th }}$ signal such that $X_{i, j}, i \neq j$ is a XPM factor and $X_{i, i}$ is a SPM factor. The nonlinear efficiency factor was calculated by numerical integration of the GN reference equation (1) in [28]. $X_{i, j}$ is a function of $\left|v_{i}-v_{j}\right|$ where $v_{i}$ and $v_{j}$ are the carrier frequencies of the $i^{\text {th }}$ and $j^{\text {th }}$ signals leading to just 80 unique values that were pre-computed. It is assumed in this work that nonlinear interference from multiple spans adds incoherently[29] and also that the SPM has been ideally compensated using digital back propagation[30] such that $X_{i, i}=0$.

\section{Network Physical Topology}

The routing, modulation, spectral assignment and launch power optimization algorithm was tested on three network topologies as described in Table 1 and shown in Fig. 1, Fig. 2 and Fig. 3. The link lengths, $Z(\mathrm{~km})$, were calculated from the great circle distance between the nodes, $Z_{G C}(\mathrm{~km})$, and a fibre routing factor using [31]

\footnotetext{
${ }^{2}$ For the DWDM channel spacing and signal symbol rate used in this work the assumption of well spaced signals and insignificant FWM was found to lead to an error of only $\approx 0.31 \%$ on the value of $X_{m}$ used in equation (7).
}

Table 1 Details of the network topologies tested.

\begin{tabular}{lllll}
\hline Name & $\begin{array}{l}\text { Nodes } \\
(\mathrm{N})\end{array}$ & $\begin{array}{l}\text { Links } \\
(\mathrm{L})\end{array}$ & $\begin{array}{l}\text { Diameter } \\
(\mathrm{km})\end{array}$ & $\begin{array}{l}\text { Avg. Node } \\
\text { Degree }\end{array}$ \\
\hline NSF Net [33] & 14 & 21 & 6000 & 3.0 \\
Google B4 [34] & 12 & 19 & 32000 & 3.2 \\
DTAG/T-Systems [35] & 14 & 23 & 1000 & 3.3 \\
\hline
\end{tabular}

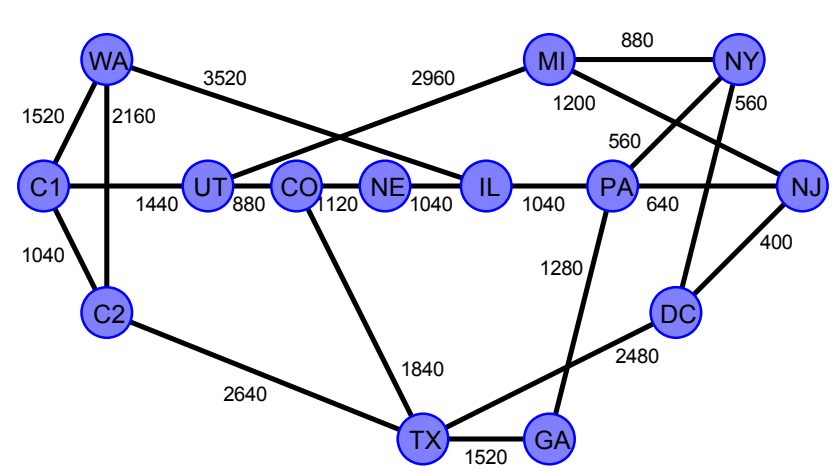

Fig. 1 The 14-node, 21-link NSF mesh topology showing the link lengths used in $(\mathrm{km})$.

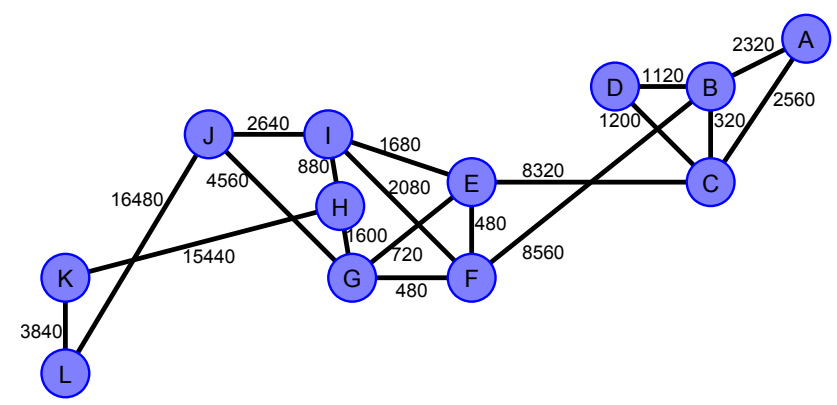

Fig. 2 The 12-node, 19-link Google B4 mesh topology showing the link lengths used in $(\mathrm{km})$.

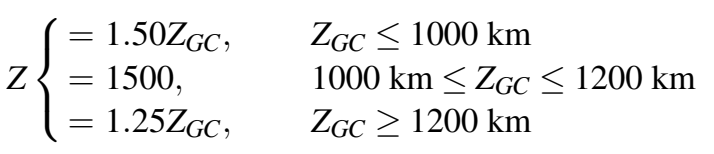

The great circle distance between the nodes was calculated from the latitude and longitude of the nodes using the Haversine formula [32].

$$
\begin{aligned}
& Z_{G C}=2 R_{e} . \\
& \arcsin \left(\sqrt{\sin ^{2}\left(\frac{\phi_{1}-\phi_{2}}{2}\right)+\cos \left(\phi_{1}\right) \cos \left(\phi_{2}\right) \sin ^{2}\left(\frac{\lambda_{1}-\lambda_{2}}{2}\right)}\right)
\end{aligned}
$$

where $R_{e}$ is the radius of the earth taken as $6367 \mathrm{~km}$ and $\phi$ and $\lambda$ are the latitude and longitude of the nodes. The link lengths were rounded to the nearest $80 \mathrm{~km}$, the span length used in this work. 


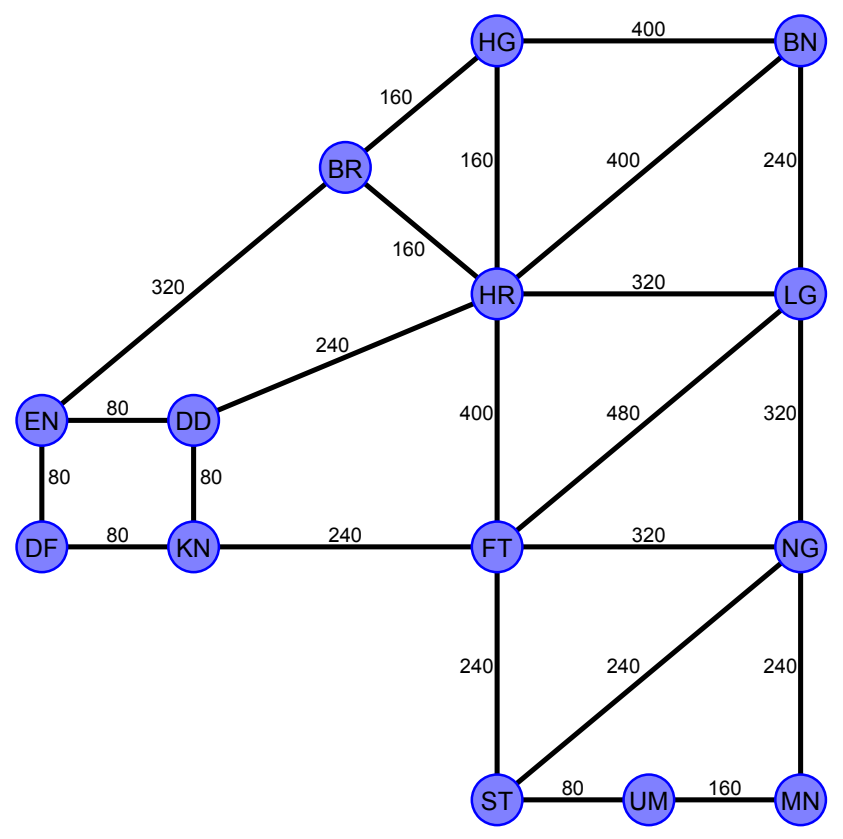

Fig. 3 The 14-node, 23-link DTAG/T-Systems National core network showing the link lengths used in $(\mathrm{km})$.

Each link was assumed to be a fibre pair consisting of uncompensated, single mode fibre in $80 \mathrm{~km}$ spans with attenuation $0.22 \mathrm{~dB} \cdot \mathrm{km}^{-1}$, chromatic dispersion $16.7 \mathrm{ps} . \mathrm{nm}^{-1} \cdot \mathrm{km}^{-1}$ and nonlinear coefficient $1.3 \mathrm{~W}^{-1} \cdot \mathrm{km}^{-1}$. The loss of each span was assumed to be ideally compensated by an EDFA with a noise figure of $5 \mathrm{~dB}$. Each fibre was assumed to support 80 DWDM channels on a $50 \mathrm{GHz}$ fixed grid, each transmitting 28 GBaud signals with a root raised cosine spectral shape (roll off 0.5 ). The ROADM nodes were assumed to be ideal, and thus not contributing to the overall noise.

The required symbol SNR for error free data transmission is shown in Table 2 for the various PM-mQAM modulation formats considered. The required symbol SNR was calculated as the theoretical symbol SNR required to give a pre-FEC BER of $4 \times 10^{-3}$ in the presence of AWGN[36, 37]. Table 2 also shows the error free data transmission rate based on the 28 GBaud symbol rate that includes a $12 \%$ overhead for FEC and signal framing.

In order to optimize the traffic throughput of a network the starting point is the assumed traffic demand profile. In this work it was assumed that the traffic demand profile was uniform and equal between all node pairs such that a normalized traffic matrix, T, was defined with the traffic between source node, $s$, and destination node, $d$, given by $T_{s, d}$ as

$T_{s, d}= \begin{cases}\frac{1}{N(N-1)} & \text { for } s \neq d \\ 0 & \text { for } s=d\end{cases}$

where $N$ is the number of nodes.
Table 2 Required symbol SNR to achieve a pre-FEC BER of $4 \times 10^{-3}$ and error free data rate, at 28 GBaud including overhead, for various PM-mQAM formats.

\begin{tabular}{lrrr}
\hline $\begin{array}{l}\text { Format } \\
\text { Acronym }\end{array}$ & $\begin{array}{r}\text { Bit } \\
\text { Loading } \\
\left(\mathrm{b} . S y m^{-1}\right)\end{array}$ & $\begin{array}{r}\text { Data } \\
\text { Rate } \\
\left(\mathrm{Gb}^{-1}\right)\end{array}$ & $\begin{array}{r}\text { Required } \\
\text { Symbol SNR } \\
(\mathrm{dB})\end{array}$ \\
\hline PM-BPSK & 2 & 50 & 5.5 \\
PM-QPSK & 4 & 100 & 8.5 \\
PM-8QAM & 6 & 150 & 12.5 \\
PM-16QAM & 8 & 200 & 15.1 \\
PM-32QAM & 10 & 250 & 18.1 \\
PM-64QAM & 12 & 300 & 21.1 \\
PM-128QAM & 14 & 350 & 23.9 \\
PM-256QAM & 16 & 400 & 26.8 \\
\hline
\end{tabular}

\section{Network Transmission Optimization}

In this section we describe the process by which the routing, channel assignment and transmitter parameters are adapted with the primary aim to maximize the network throughput and secondary aims to minimize the number of transceivers and maximize the available SNR margin above that required for error free transmission. An overall flow diagram illustrating the process is shown in Fig. 4.

A pre-solve stage calculates the k-shortest routes between each node pair, the SNR of each route and selects suitable modulation formats. Next a mixed integer linear program, MILP, is used to allocate transmitters to routes and DWDM channels to maximize the network throughput within the constraint of the traffic profile and physical resources. Having found the maximum network throughput the MILP is resolved with the secondary objective to minimize the number of transmitters, minimize nonlinear interference and group highly interfering DWDM channels. These processes are described in detail in Sect. 4.1.

Given a network solution with transmitters allocated to routes and DWDM channels the next step is to optimize individual launch powers and assign spectrum to each DWDM channel. The highly interfering channels have been grouped to low DWDM channel numbers so the DWDM channels are not allocated sequential spectral slots but are dispersed to reduce the nonlinear interference. The process of assigning DWDM channels to physical spectrum and optimizing individual launch powers is described in Sect. 4.2. The aim of this process is to maximize the available SNR margin given a network solution developed in Sect. 4.1.

Where the process of spectral assignment and launch power optimization reveals a large SNR margin the initial pre-solve stage is re-calculated on the temporary assumption that the modulation formats required SNR can be reduced, allowing higher order modulation on some routes than would initially have been allowed. The MILP, launch power optimization and spectral assignment are repeated to obtain new network solutions with potentially higher 
throughput but lower SNR margin. Provided the SNR margin is $>0$ the new network solution was deemed viable.

\subsection{Static Routing, Modulation and Channel Assignment}

This section describes the approach used to optimize the routing, modulation format and channel assignment when the launch power and nonlinear interference are precalculated and fixed. We leave the spectral assignment of the channels and launch power allocation to Sect. 4.2. The primary aim of this optimization was to maximize the network traffic throughput, with a secondary aim of minimizing the number of transmitters and minimizing the nonlinear interference noise for robust transmission. In order to remove the launch power consideration from this stage of the optimization the launch power was pre-optimized by setting a constant and equal optimized power and similar to the LOGON[38] strategy all the DWDM channels were considered fully occupied with a constant power. This assumption removes the exact routing and DWDM channel allocation from the SNR estimation, decoupling the choice of modulation format from the routing and channel allocation. The symbol SNR of all the channels over a route was taken as the minimum SNR, $S N R^{M}$, on the worst case, central, channel such that

$S N R^{M}=\frac{p_{0}}{N_{s} n_{A S E}+N_{s} X_{m} p_{0}^{3}}$

where $p_{0}$ is the equal launch power of all the channels, $N_{S}$ is the number of fibre spans the signal traverses, $n_{A S E}$ is the ASE noise accumulated in a single fibre span and $X_{m}=\max _{j} \sum_{i} X_{i, j}$, is the maximum nonlinear interference efficiency for a fully loaded single span. The launch power, $p_{0}$, is optimized to maximize the SNR of equation (7). The optimum launch power is independent of the route length since both noise terms depend linearly on the number of spans here. For the fibre, EDFA and signal parameters used, the ASE noise accumulated from each fibre span, $n_{A S E}$, was $0.00065 \mathrm{~mW}$, and the maximum nonlinear interference factor, $X_{m}$, was $0.00067 \mathrm{~mW}^{-2}$ such that the optimum launch power was $0.79 \mathrm{~mW}(-1.0 \mathrm{dBm})$. The worst case SNR, $S N R^{M}$, of each route can be simply calculated given the number of fibre spans traversed.

To solve the routing and spectral allocation problem there are two widespread integer linear programming, ILP, formulations; those based on flow and those based on routes $[39,40]$. Since the transmission SNR for a given connection depends on the overall route, and not on individual links, the modulation format will be route-dependent and the routebased ILP formulation is more appropriate here.

As a preliminary stage to solving the ILP problem, first the k-shortest routes[41], $k=25$, between each sourcedestination node pair were calculated and the $S N R^{M}$ of each

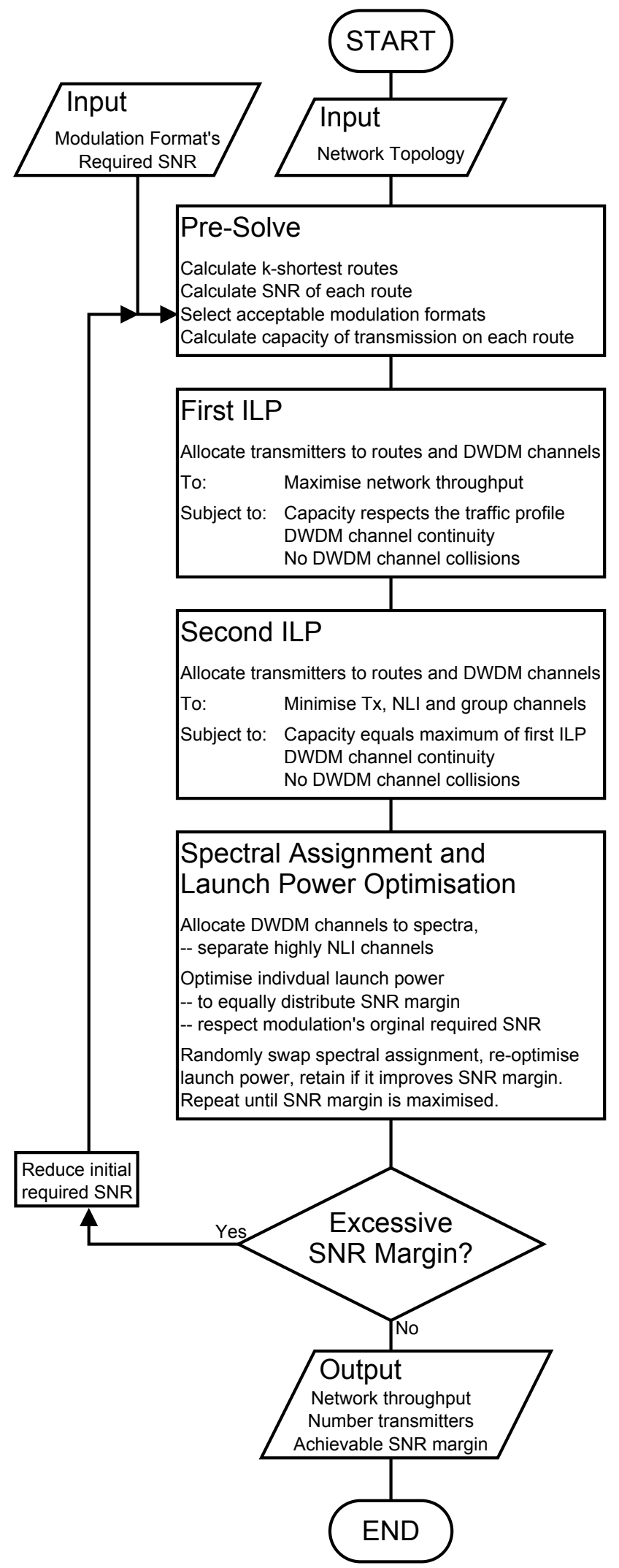

Fig. 4 Flow diagram to illustrate the overall network optimisation process. 
route was calculated based on the number of fibre spans in the route as equation (7) assuming all DWDM channels are occupied with a signal at the optimized power. The modulation formats where the route SNR exceeds the modulation formats required SNR were chosen for each route from the modulation formats listed in Table 2 and the capacity of each route with each modulation format was calculated.

Next, the allocation of DWDM channels to routes was optimized as a mixed integer linear program, MILP, in order to maximize the traffic throughput. It should be noted that the traffic matrix is symmetric, $T_{s, d}=T_{d, s}$, and the network is symmetric with fibre pairs, so as is customary we reduced the problem by solving for $d>s$ only. In order to describe the MILP problem the following notation was used

\section{Constants:}

- $N$ : number of nodes,

- L: number of links,

- $K$ : number of shortest routes,

- $W$ : number of DWDM channels,

- $M$ : number of modulation formats available over a given route $r_{s, d, k}$,

Parameters:

- $s, d$ : the source and destination nodes $\in\{1,2, \ldots, N\}$,

- $l:$ a link $\in\{1,2, \ldots, L\}$,

- $k$ : a shortest route $\in\{1,2, \ldots, K\}$,

- $w$ : a DWDM channel $\in\{1,2, \ldots, W\}$,

- $m$ : a modulation format $\in\{1,2, \ldots, M\}$,

- $T_{s, d}$ : the normalized traffic demand between source, $s$, and destination,$d$,

- $r_{s, d, k}$ : the $k^{\text {th }}$ shortest route between source, $s$, and destination,$d$,

- $\delta_{s, d, k, l}^{L}$ : is set to 1 if route $r_{s, d, k}$ traverses link $l, 0$ otherwise,

- $S N R_{s, d, k}^{M}$ : the worst case symbol SNR for transmission over route $r_{s, d, k}$,

- $C_{s, d, k, m}$ : the capacity of transmission over route $r_{s, d, k}$ with modulation format, $m$.

- $S N R_{s, d, k, m}^{R}$ : the required symbol SNR for error free transmission over route $r_{s, d, k}$ at the capacity $C_{s, d, k, m}$,

Variables:

- $c$ : the total throughput of the network, a multiplying factor to the normalized traffic matrix $\mathbf{T}$ to define the actual traffic demand,

- $\delta_{s, d, k, m, w}^{F}$ : is 1 if a transceiver for route $r_{s, d, k}$ uses modulation format, $m$, and DWDM channel $w, 0$ otherwise.

The aim is to optimize $c$ as a continuous variable and $\delta_{s, d, k, m, w}^{F}$ as binary variables, so that

$c_{\max }=\max _{c, \delta_{s, d, k, m, w}^{F}} c$ subject to the total capacity between all source, $s$, and destination, $d$, nodes exceeding the demand

$$
\sum_{w} \sum_{m} \sum_{k} \delta_{s, d, k, m, w}^{F} C_{s, d, k, m}-c T_{s, d}>=0 \quad \forall \quad s, d>s .
$$

and the number of signals occupying DWDM channel, $w$, in any link, $l$, does not exceed 1 ,

$\sum_{m} \sum_{k} \sum_{s} \sum_{d>s} \delta_{s, d, k, m, w}^{F} \delta_{s, d, k, l}^{L}<=1 \quad \forall \quad l, w$.

The ILP optimization was solved using IBM CPLEX® and for the results presented in this paper the computation took typically a few hours on a dual quad core computer with 16 threads running at $2.3 \mathrm{GHz}$.

Given that the maximum network throughput $c_{\max }$ has been calculated the MILP was re-solved with the secondary objective to group highly interfering channels, for later separation, while minimizing the total number of transmitters and the overall nonlinear interference. Optimize $\delta_{s, d, k, m, w}^{F}$ as binary variables to

$$
\begin{aligned}
\underset{\delta_{s, d, k, m, w}^{F}}{\min } & \sum_{w} \sum_{m} \sum_{k} \sum_{s} \sum_{d>s} \delta_{s, d, k, m, w}^{F} . \\
& {\left[1000+\frac{Z_{s, d, k}}{10000}\left(\frac{S N R_{s, d, k, m}^{R}}{S N R_{s, d, k}^{M}}\right)^{2}\left(\frac{w}{10}+100\right)\right] }
\end{aligned}
$$

subject to the constraints of equations (9) and (10) where $c$ has been replaced by $c_{\text {max }}$. The large constant in the objective function ensures that the minimum number of transmitters are used. The term $\frac{S N R_{s, d, k, m}^{R}}{S N R_{s, d, k}^{M}}$ is the ratio of the modulation formats' required SNR to the basic SNR of the route used in the routing stage, the worst case where all channels are occupied. This ratio is related to the expected transmitter power thus the square multiplied by the route length $Z_{s, d, k}$ denotes the channels likelihood to cause nonlinear interference. By dividing $Z_{s, d, k}$ by 10000 a constant of the order of the maximum of $Z_{s, d, k}$ the weight term $\frac{Z_{s, d, k}}{10000}\left(\frac{S N R_{s, d, k, m}^{R}}{S N R_{s, d, k}^{M}}\right)^{2}$ is of order 1. By weighting the objective function with the channel number, more highly interfering signals will be placed in the lowest available channel number, thus grouping them together. The constant added to the channel weight ensures that nonlinear interference is always more weighted than channel number. The objective function weight increases by order 1000 for additional transmitters, order $<100$ for nonlinear interference and order $<10$ for higher channel numbers. So this objective function tries to, in order of priority, minimize the number of transmitters, then minimize the nonlinear interference and finally group highly interfering signals. There is still some considerable scope to improve this objective function to more closely achieve these stated aims. 


\subsection{Launch Power and Spectral Assignment}

The nonlinear interference noise equation (3) was developed with point-to-point links in mind however it applies equally well to mesh networks. The $i^{\text {th }}$ or $j^{\text {th }}$ transmitter-receiver pair each corresponds to one active $\{s, d, k, m, w\}$ connection between source, $s$, destination, $d$, by the $k^{\text {th }}$ shortest route, with modulation format, $m$, and utilizing wavelength channel $w$ where $\delta_{s, d, k, m, w}^{F}=1$. For the $i^{\text {th }}$ transmitter in a mesh network the ASE noise, $n_{A S E, i}$ is given by

$n_{A S E, i}=10^{\frac{N F}{10}} h v R \sum_{l}\left[\delta_{i, l}^{L} N_{s, l} 10^{\frac{A_{s p a n}}{10}}\right]$

where $N_{s, l}$ is the number of spans in link $l$ and $\delta_{i, l}^{L}$ is 1 if the signal from the $i^{\text {th }}$ transmitter-receiver pair traverses link $l$, zero otherwise. Similarly the nonlinear interference is given by a summation of the nonlinear interference accumulated on the $i^{\text {th }}$ transmitted signal as it traverses each link on its route, thus the accumulated nonlinear efficiency, $X_{i, j}^{A}$, is given by

$X_{i, j}^{A}=\sum_{l}\left[\delta_{i, l}^{L} \delta_{j, l}^{L} N_{s, l} X\left(\left|v_{i}-v_{j}\right|\right)\right]$

where $X(\Delta v)$ is the single span efficiency for the nonlinear interference between signals spaced by a frequency, $\Delta v$. Given that the $i^{\text {th }}$ transmitter-receiver pair requires a symbol SNR, $S N R_{i}^{R}$, with an overall margin $S N R_{\text {margin }}$ then the launch powers, $\mathbf{p}$ should be minimized to achieve a symbol SNR of $S N R_{\text {margin }} \mathbf{S N R}^{\mathbf{R}}$. The launch powers were iteratively optimized to achieve the required SNR and were initialized based on ASE noise alone using

$p_{i}=S N R_{\text {margin }} S N R_{i}^{R} n_{A S E, i}$.

For a given network state with launch powers, $\mathbf{p}$ the SNR of the $i^{\text {th }}$ signal is given by,

$S N R_{i}=\frac{p_{i}}{n_{A S E, i}+p_{i} \sum_{j}\left(X_{i, j}^{A} p_{j}^{2}\right)}$.

The variation of the SNR with launch power around the current network state, $\frac{\mathbf{d S N R}}{\mathbf{d p}}$ is given by

$\frac{\mathrm{d} S N R_{i}}{\mathrm{~d} p_{j}}=S N R_{i}^{2}\left[\frac{n_{A S E, i=j}}{p_{i}^{2}}-2 X_{i, j}^{A} p_{j}\right]$

where $n_{A S E, i=j}=n_{A S E, i}$ for $i=j$, zero otherwise. The iteration update is given by

$\Delta \mathbf{p}=\left(S N R_{\text {margin }} \mathbf{S N R}^{\mathbf{R}}-\mathbf{S N R}\right)\left(\frac{\mathbf{d S N R}}{\mathbf{d p}}\right)^{-1}$

In order to improve the speed of convergence and dampen oscillations in the computation the iteration update was restricted at each step by multiplying by $\mu<1$ where

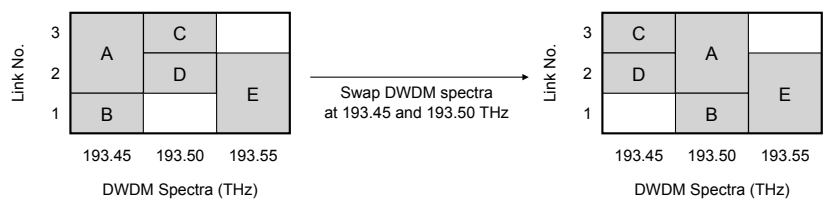

Fig. 5 Illustration of DWDM spectrum swapping process. A-E refer to transmitted signals, grey block are occupied link-spectral slots. After swapping the spectral continuity and collision free constraints are maintained.

$\mu= \begin{cases}\min _{i}\left(\frac{0.1}{\Delta P_{i}}\right) & \text { for } \min _{i}\left(\frac{0.1}{\Delta P_{i}}\right)<1 \\ 1 & \text { otherwise }\end{cases}$

the launch powers, $\mathbf{p}$ were then updated as

$\mathbf{p}:=\mathbf{p}+\mu \Delta \mathbf{p}$

the iteration proceeds through equations (15) to (19) until the launch powers, $\mathbf{p}$, converge. The algorithm was found to converge within 20 iterations for $\approx 600$ transmitters provided the required SNR with margin can be achieved. The SNR margin was then increased incrementally until the iteration would no longer converge and no launch power solution could be found. The maximum achievable margin was taken as the largest margin for which a solution to the power optimization was possible.

The DWDM channels as obtained from the routing modulation and channel assignment ILP were then assigned to DWDM spectra. The separation of higher power signals has been shown[15] to reduce the overall interference. The assignment was initial done manually to separate the lower channel numbers by assigning them well separated spectra. Since we are considering a fixed grid, each DWDM channel from the solution of Sect. 4.1, is self-contained, there is no spectral contiguity constraint. This allows all of the transmitters operating in a given DWDM channel to be moved to another spectra assignment without disturbing the spectral continuity or collision constraints. Thus we can swap all of the transmitters in one DWDM channel with another DWDM channel and this possibility was used to improve the DWDM channel spectral assignment. The process is illustrated in Fig. 5 for a simple network with three links and three DWDM channels. Thus the manual assignment was improved by swapping random pairs of DWDM channels and calculating the achievable margin by following the iterative launch power optimization described above. The new DWDM spectra assignment was then retained if this improved the achievable margin or returned to the original spectral positions if it did not. Random pairs were swapped until no further improvement in achievable margin could be made.

For the NSF network utilizing a fixed PM-QPSK modulation format the initial grouped channel assignments from 
Fixed modulation format, fixed power, worst case Adapted modulation format, optimized Power
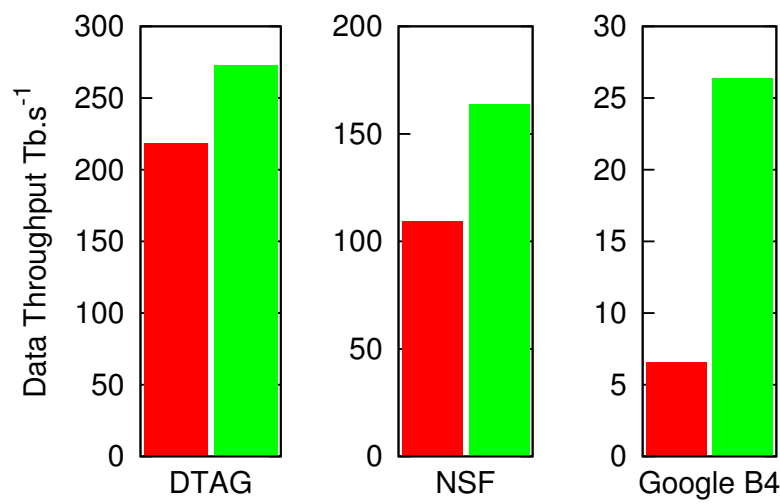

Fig. 6 Total launch power across all transmitters in each DWDM channel for the NSF network for the fixed PM-QPSK modulation solution. Initial grouped DWDM channel assignments, giving $2.6 \mathrm{~dB}$ SNR margin.

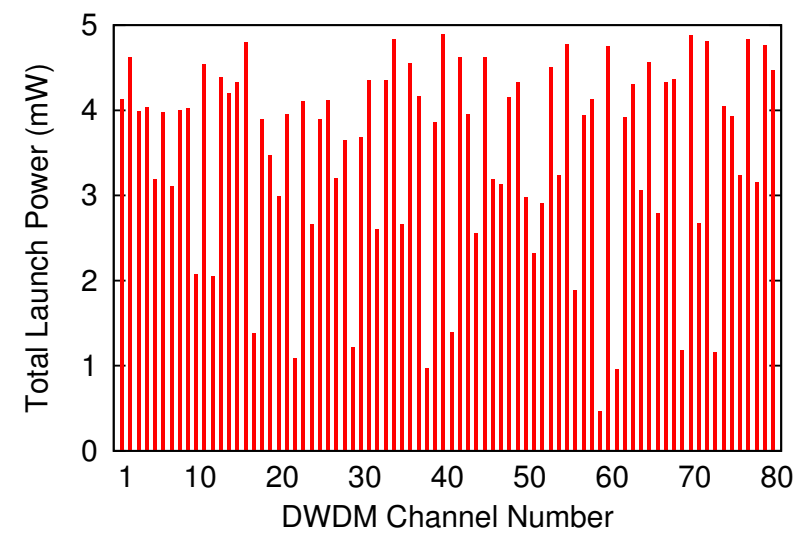

Fig. 7 Total launch power across all transmitters in each DWDM channel for the NSF network for the fixed PM-QPSK modulation solution. Optimal DWDM channel assignments, giving $3.3 \mathrm{~dB}$ SNR margin.

the ILP solution give an achievable SNR margin of $2.6 \mathrm{~dB}$. Fig. 6 shows the total launch power, summed over all transmitters, in each DWDM channel after the individual launch powers have been optimized as described above but retaining the grouped channel assignments. Following spectral assignment the achievable SNR margin was increased to $3.3 \mathrm{~dB}$ and Fig. 7 shows the total launch power, summed over all transmitters, in each DWDM channel, clearly showing that high power channels have been spectrally separated.

\section{Results from Example Networks}

For each of the three example networks, we consider an initial baseline optimization for a fixed modulation format, fixed launch power and assumed full channel occupancy. The fixed modulation format was chosen as the highest order modulation format that can be transmitted error free be- tween the furthest spaced nodes. The SNR margin of this fixed modulation format solution was improved by optimizing the spectral assignment and individual launch powers and showed that by moving away from the fixed power full occupancy assumption a considerable SNR margin was available for improving the network throughput. The networks were then solved for adaptive modulation formats and finally with reduced initial SNR requirements.

Beginning with the NSF network[33] of Fig. 1, under the assumption of equal launch power and fully loaded links the SNR of the longest shortest-path route allows PM-QPSK modulation. Thus PM-QPSK is the baseline traditional case for this network as it is suitable for transmission between all node pairs. By optimizing the routing and channel allocation using the ILP described in Sect. 4.1 while maintaining the fixed PM-QPSK modulation format a network throughput of $109.2 \mathrm{~Tb}^{-1} \mathrm{~s}^{-1}$ was achieved utilizing 1092 transmitters and with a worst case SNR margin of $1.6 \mathrm{~dB}$. Taking this fixed PM-QPSK routing solution and optimizing the launch power and spectral allocation, as described in Sect. 4.2, a SNR margin of $3.3 \mathrm{~dB}$ was possible.

It is then the aim to utilize this margin to increase the network throughput. First the modulation format was adapted to the route by allowing the ILP of Sect. 4.1 the choice of all modulation formats on each route that have a required SNR less than the route worst case SNR calculated based on equal launch powers and fully loaded links. The adaptation of the modulation format increased the network throughput to $127.4 \mathrm{~Tb}^{-1} \mathrm{~s}^{-1}$ utizing 992 transmitters. Following optimization of the launch power and spectral allocation a SNR margin of $2.3 \mathrm{~dB}$ was achieved. To further access this margin the modulation formats required SNR were temporarily reduced by $1.6 \mathrm{~dB}$ during the initial routing, modulation and channel assignment. This allowed a network throughput of $163.8 \mathrm{~Tb}^{-1} \mathrm{~s}^{-1}$ utilizing 1040 transmitters and following launch power and spectral allocation achieved a SNR margin of $0.1 \mathrm{~dB}$.

Thus by optimizing the routing, modulation, launch power and spectral assignment the throughput has been increased by $50 \%$, for the NSF network, over the baseline traditional route independent case. Thus the network throughput has been increased by increasing the overall spectral efficiency at the expense of the SNR margin. These results are summarized in Table 3.

Considering the much longer Google B4 network[34] of Fig. 2 under the assumption of equal launch power and fully loaded links it is not possible to transmit the lowest order modulation format, PM-BPSK across the longest shortestpath route. In order to achieve a SNR suitable for PM-BPSK on the longest shortest route under the assumption of equal launch power and fully loaded links the channel spacing was increased to $200 \mathrm{GHz}$ and the number of channels reduced to 20 . By optimizing the routing and channel allocation a base- 
Table 3 Summary of results for the NSF network.

\begin{tabular}{llll}
\hline Optimization & $\begin{array}{l}\text { Network } \\
\text { Throughput } \\
\left(\text { Tb.s }^{-1}\right)\end{array}$ & $\begin{array}{l}\text { Number } \\
\text { Transmitters }\end{array}$ & $\begin{array}{l}\text { SNR } \\
\text { Margin } \\
(\mathrm{dB})\end{array}$ \\
\hline $\begin{array}{l}\text { Fixed PM-QPSK } \\
\text { Equal Power }\end{array}$ & 109.2 & 1092 & 1.6 \\
Fully loaded links & & 1092 & 3.3 \\
$\begin{array}{l}\text { Fixed PM-QPSK } \\
\text { Optimized Power } \\
\text { and channel spectrum }\end{array}$ & 109.2 & & \\
$\begin{array}{l}\text { Adapted PM-mQAM } \\
\text { Optimized Power } \\
\text { and channel spectrum }\end{array}$ & 127.4 & 992 & 2.3 \\
$\begin{array}{l}\text { Adapted PM-mQAM } \\
\text { Optimized Power } \\
\text { and channel spectrum }\end{array}$ & 163.8 & 1040 & 0.1 \\
\hline
\end{tabular}

line network throughput of $6.6 \mathrm{~Tb} . \mathrm{s}^{-1}$ was achieved utilizing 132 transmitters with a worst case SNR margin of $0.2 \mathrm{~dB}$. Taking this fixed PM-BPSK routing solution and optimizing the launch power and spectral assignment a SNR margin of $3.3 \mathrm{~dB}$ was possible.

The limited number of channels leads to just one bidirectional connection between each node pair providing just $50 \mathrm{~Gb}^{-1}$, in each direction, between each node pair. Given that not all the channels will be occupied and that occupied channels can be separated in the launch power and spectral assignment process the channel spacing was reduced to $100 \mathrm{GHz}$ to provide 40 channels to improve the granularity of the routing solution. In order to connect the furthest spaced nodes the modulation formats required SNR were temporarily reduced by $1.3 \mathrm{~dB}$ during the initial routing, modulation and channel assignment to allow PM-BPSK over the longest shortest-path route under the fully loaded links worst case. A network throughput of $13.2 \mathrm{~Tb}^{-\mathrm{s}^{-1}} \mathrm{utiliz}^{-}$ ing 264 transmitters was achieved and following optimizing of the launch power and spectral assignment a SNR margin of $1.7 \mathrm{~dB}$ was possible.

To further improve the network throughput the modulation format was adapted to the route by allowing the ILP of Sect. 4.1 the choice of all modulation formats on each route that have a required SNR, that is temporarily reduced by $1.3 \mathrm{~dB}$, less than the route worst case SNR calculated based on equal launch powers and fully loaded links. This allowed a network throughput of $26.4 \mathrm{~Tb}^{-1}{ }^{-1}$ utilizing 300 transmitters and following launch power and spectral assignment optimization a margin just above $0.0 \mathrm{~dB}$ was achieved.

Thus by optimizing the routing, modulation, spectral assignment and launch power the throughput has been increased by $300 \%$, for the Google B4 network, over the baseline traditional route independent case. The reason for these
Table 4 Summary of results for the Google B4 network.

\begin{tabular}{llll}
\hline Optimization & $\begin{array}{l}\text { Network } \\
\text { Throughput } \\
\left(\mathrm{Tb}_{\mathrm{s}}{ }^{-1}\right)\end{array}$ & $\begin{array}{l}\text { Number } \\
\text { Transmitters }\end{array}$ & $\begin{array}{l}\text { SNR } \\
\text { Margin } \\
(\mathrm{dB})\end{array}$ \\
\hline $\begin{array}{l}\text { Fixed PM-BPSK } \\
\text { 200 GHz grid }\end{array}$ & 6.6 & 132 & 0.2 \\
$\begin{array}{l}\text { Equal Power } \\
\text { Fully loaded links }\end{array}$ & & & \\
$\begin{array}{l}\text { Fixed PM-BPSK } \\
\text { 200 GHz grid }\end{array}$ & 6.6 & 132 & 3.3 \\
$\begin{array}{l}\text { Optimized Power } \\
\text { and channel spectrum }\end{array}$ & & & \\
$\begin{array}{l}\text { Fixed PM-BPSK } \\
\text { 100 GHz grid }\end{array}$ & 13.2 & 264 & 1.7 \\
$\begin{array}{l}\text { Optimized Power } \\
\text { and channel spectrum }\end{array}$ & & & \\
$\begin{array}{l}\text { Adapted PM-mQAM } \\
100 \text { GHz grid } \\
\text { Optimized Power } \\
\text { and channel spectrum }\end{array}$ & 26.4 & 300 & 0.0 \\
\hline
\end{tabular}

gains are twofold, firstly the increase in spectral efficiency at the expense of SNR margin and secondly the unblocking achieved by the reduction of granularity by allowing an increased number of channels. These results are summarized in Table 4.

Finally we consider the shorter DTAG/T-Systems National core network[35] of Fig. 3, under the assumption of equal launch power and fully loaded links the SNR of the longest shortest-path route allows PM-16QAM modulation. Thus PM-16QAM is the baseline traditional case for this network as it is suitable for transmission between all node pairs. By optimizing the routing and channel allocation using the ILP described in Sect. 4.1 while maintaining the fixed PM-16QAM modulation format a network throughput of 218.4 Tb.s ${ }^{-1}$ was achieved utilizing 1092 transmitters and with a worst case SNR margin of $2.8 \mathrm{~dB}$. Taking this fixed PM-16QAM routing solution and optimizing the launch power and spectral allocation, as described in Sect. 4.2, a SNR margin of $4.3 \mathrm{~dB}$ was possible.

Allowing the ILP of Sect. 4.1 the choice of all modulation formats on each route that have a required SNR less than the route SNR calculated based on equal launch powers and fully loaded links was too complex in this case due to the larger number of formats given the higher overall SNR for this shorter network. In order to reduce the complexity the lower order formats, PM-BPSK and PM-QPSK were removed from the choice as these should not be used given the constraint to minimize the number of transmitters. The adaptation of the modulation format increased the network throughput to $273.0 \mathrm{~Tb}^{-1}{ }^{-1}$ utilizing 1002 transmitters. Following optimization of the launch power and spectral allo- 
Table 5 Summary of results for the DTAG/T-Systems National core network.

\begin{tabular}{llll}
\hline Optimization & $\begin{array}{l}\text { Network } \\
\text { Throughput } \\
\left(\mathrm{Tb}^{-1}{ }^{-1}\right)\end{array}$ & $\begin{array}{l}\text { Number } \\
\text { Transmitters }\end{array}$ & $\begin{array}{l}\text { SNR } \\
\text { Margin } \\
(\mathrm{dB})\end{array}$ \\
\hline $\begin{array}{l}\text { Fixed PM-16QAM } \\
\text { Equal Power }\end{array}$ & 218.4 & 1092 & 2.8 \\
$\begin{array}{l}\text { Fully loaded links } \\
\text { Fixed PM-16QAM } \\
\text { Optimized Power } \\
\text { and channel spectrum }\end{array}$ & 218.4 & 1092 & 4.3 \\
$\begin{array}{l}\text { Adapted PM-mQAM } \\
\text { Optimized Power } \\
\text { and channel spectrum }\end{array}$ & 273.0 & 1002 & 1.1 \\
\hline
\end{tabular}

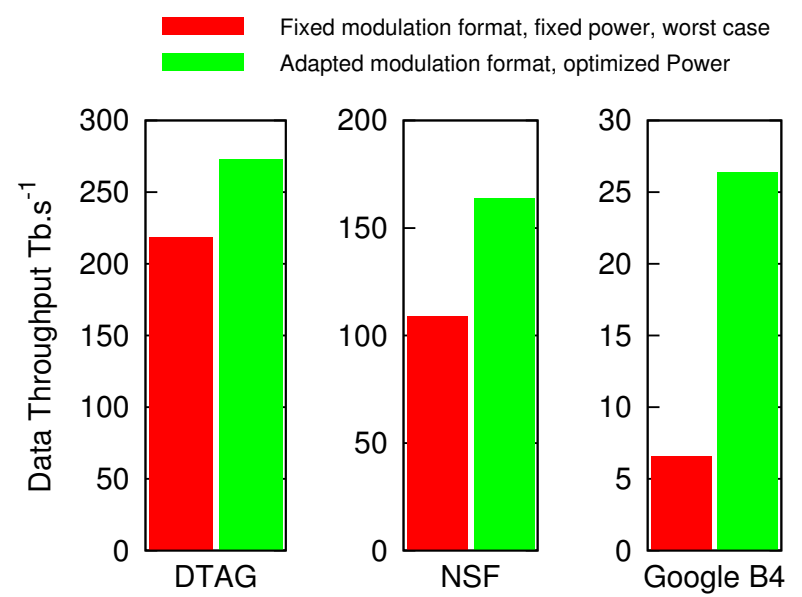

Fig. 8 Comparison of network throughput for the baseline fixed modulation fixed power worst case fully loaded links case and the adapted modulation adapted power case. For the three network topologies tested.

cation a SNR margin of $1.1 \mathrm{~dB}$ was achieved. This margin could not be used to further increase the network throughput. The granularity of the network solutions is such that the next higher throughput solution has a SNR margin less than $0.0 \mathrm{~dB}$ and did not allow error free transmission.

Thus by optimizing the routing, modulation, spectral assignment and launch power the throughput has been increased by $25 \%$, for the DTAG/T-Systems Nation core network, over the baseline traditional route independent case. These results are summarized in Table 5.

Fig. 8 summarizes the network throughput for each of the three test topologies for the baseline and optimized case. We have shown that for each of these three network topologies, with quite different diameters, the optimization of the launch power and channel spectra assignment leads to a SNR margin of around 3-4 $\mathrm{dB}$ for the fixed go anywhere baseline modulation format. This margin can be traded to increase the average modulation format order by approximately $1 \mathrm{~b} \cdot \mathrm{s}^{-1} \cdot \mathrm{Hz}^{-1} \cdot \mathrm{pol}^{-1}$ increasing the network throughput. For the smallest diameter DTAG/TSystems National core network where the fixed modulation format is PM-16QAM, 4 b.s ${ }^{-1} \cdot \mathrm{Hz}^{-1} \cdot \mathrm{pol}^{-1}$, the increase should give a $25 \%$ improvement in throughput. For the NSF network where the fixed modulation format is PM-QPSK, $2 \mathrm{~b} \cdot \mathrm{s}^{-1} \cdot \mathrm{Hz}^{-1} \cdot \mathrm{pol}^{-1}$, the increase should give a $50 \%$ improvement in network throughput and finally for the Google B4 network where the fixed modulation format is PM-BPSK, $1 \mathrm{~b} \cdot \mathrm{s}^{-1} \cdot \mathrm{Hz}^{-1} \cdot \mathrm{pol}^{-1}$, the increase should give a $100 \%$ improvement in network throughput. This is approximately what was observed except in the Google B4 network where an increase in throughput of $300 \%$ was achieved. It is believed the additional increase in network throughput is caused by unblocking in this network where the integer granularity on the number of transmitters can be a significant constraint to throughput. Initially with just 20 channels the topological bottleneck allows just one transmitter to operate between each node pair. By increasing the number of channels to 40 with a fixed PM-BPSK modulation format, 2 transmitters can operate between each node pair although the topological bottleneck would allow 2.5. The adaptation of the modulation format allows fewer transmitters to provide the capacity over the short connections and releases bandwidth for the long connections, this allows the average number of transmitters between each node pair to approach 2.5 providing fuller utilization of the available bandwidth. This is similar to the higher gains observed for unblocking in flexible networks[42].

\section{Conclusions}

We have presented an algorithm to maximize the network throughput through static optimization of routing, modulation format, spectral assignment and launch power allocation within a nonlinear impaired optical mesh network. From a baseline case defined for fixed launch power, fully loaded channels with a fixed modulation format, suitable for the longest shortest-path route, the gains in network throughput for three example mesh networks have been quantified. For the DTAG, NSF and Google B4 network topologies an improvement of $25 \%, 50 \%$ and $300 \%$ in network throughput respectively was achieved.

We show that the SNR margin can be increased through the optimization of channel spectral assignment and launch power and that the SNR margin can be traded for network capacity by adapting the modulation format to the route. The increase in network throughput can be attributed to two effects, the overall increase in spectral efficiency achieved as a result of the trade off against SNR margin and through an unblocking of network bottlenecks by utilizing less bandwidth for the shorter connections releasing bandwidth for the longer connections. The second effect is more signif- 
icant in networks where there are few transmitter-receiver pairs connecting each node pair.

The optimization of routing, modulation format, spectral assignment and launch power allocation described here is a multistage process, first to optimize the routing, modulation format and channel assignment and then to maximize the SNR margin by optimizing the channel spectral assignment and launch power. Where sufficient SNR margin is available the initial stage can be repeated to increase network throughput. This multistage process results in good but potentially sub-optimal results. An important avenue for future study would be to combine these stages to provide a single optimization process. In future work, it is also intended to include the benefits of improved spectral efficiency through the use of a flexible grid.

\section{References}

1. R.M. Schmogrow, D. Hillerkuss, M. Dreschmann, M. Huebner, M. Winter, J. Meyer, B. Nebendahl, C. Koos, J. Becker, W. Freude, J. Leuthold: Real-Time Software-Defined Multiformat Transmitter Generating 64QAM at 28 GBd. IEEE Photonics Technology Letters 22(21), 1601 (2010). DOI 10.1109/LPT.2010.2073698

2. H.Y. Choi, T. Tsuritani, I. Morita: BER-adaptive flexible-format transmitter for elastic optical networks. Optics Express 20(17), 18652 (2012). DOI 10.1364/OE.20.018652

3. K. Roberts, C. Laperle: Flexible Transceivers. in 38th European Conference and Exposition on Optical Communications, 1, p. We.3.A.3 (Amsterdam (NL), 2012) (2012). DOI 10.1364/ECEOC.2012.We.3.A.3

4. D.J. Ives, P. Bayvel, S.J. Savory: Physical Layer Transmitter and Routing Optimization to Maximize the Traffic Throughput of a Nonlinear Optical Mesh Network. in International Conference on Optical Network Design and Modeling, pp. 168-173 (Stockholm (SE), 2014) (2014)

5. A. Nag, M. Tornatore, B. Mukherjee: Optical Network Design With Mixed Line Rates and Multiple Modulation Formats. Journal of Lightwave Technology 28(4), 466 (2010). DOI 10.1109/JLT.2009.2034396

6. M. Jinno, H. Takara, B. Kozicki, Y. Tsukishima, Y. Sone, S. Matsuoka: Spectrum-efficient and scalable elastic optical path network: architecture, benefits, and enabling technologies. IEEE Communications Magazine 47(11), 66 (2009). DOI 10.1109/MCOM.2009.5307468

7. O. Gerstel, M. Jinno, A. Lord, S.J.B. Yoo: Elastic optical networking: a new dawn for the optical layer? IEEE Communications Magazine 50(2), s12 (2012). DOI 10.1109/MCOM.2012.6146481

8. B. Kozicki, H. Takara, Y. Sone, A. Watanabe, M. Jinno: DistanceAdaptive Spectrum Allocation in Elastic Optical Path Network ( SLICE ) with Bit per Symbol Adjustment. in Optical Fiber Communication Conference, p. OMU3 (San Diego, CA. (USA), 2010) (2010)

9. K. Christodoulopoulos, I. Tomkos, E.A. Varvarigos: Elastic Bandwidth Allocation in Flexible OFDM-Based Optical Networks. Journal of Lightwave Technology 29(9), 1354 (2011). DOI 10.1109/JLT.2011.2125777

10. E. Palkopoulou, M. Angelou, D. Klonidis, K. Christodoulopoulos, A. Klekamp, F. Buchali, E. Varvarigos, I. Tomkos: Quantifying Spectrum, Cost, and Energy Efficiency in Fixed-Grid and FlexGrid Networks [Invited]. Journal of Optical Communications and Networking 4(11), B42 (2012). DOI 10.1364/JOCN.4.000B42
11. F. Forghieri, R. Tkach, A. Chraplyvy, D. Marcuse: Reduction of four-wave mixing crosstalk in WDM systems using unequally spaced channels. IEEE Photonics Technology Letters 6(6), 754 (1994). DOI 10.1109/68.300184

12. A. Adhya, D. Datta: Design methodology for WDM backbone networks using FWM-aware heuristic algorithm. Optical Switching and Networking 6(1), 10 (2009). DOI 10.1016/j.osn.2008.05.006

13. A. Nag, M. Tornatore, B. Mukherjee: Power Management in Mixed Line Rate Optical Networks. in Integrated Photonics Research, Silicon and Nanophotonics and Photonics in Switching, 2, p. PTuB4 (OSA, Monterey, CA. (USA), 2010) (2010). DOI 10.1364/PS.2010.PTuB4

14. H. Beyranvand, J.a. Salehi: A Quality-of-Transmission Aware Dynamic Routing and Spectrum Assignment Scheme for Future Elastic Optical Networks. Journal of Lightwave Technology 31(18), 3043 (2013). DOI 10.1109/JLT.2013.2278572

15. D.J. Ives, S.J. Savory: Transmitter Optimized Optical Networks. in National Fiber Optic Engineers Conference, p. JW2A.64 (OSA, Anaheim, CA. (USA), 2013) (2013). DOI 10.1364/NFOEC.2013.JW2A.64

16. D. Rafique, A.D. Ellis: Nonlinear Penalties in Dynamic Optical Networks Employing Autonomous Transponders. IEEE Photonics Technology Letters 23(17), 1213 (2011). DOI 10.1109/LPT.2011.2158603

17. A. Splett, C. Kurtske, K. Petermann: Ultimate transmission capacity of amplified optical fiber communication systems taking into account fiber nonlinearities. in European Conference on Optical Communications, p. MoC2.4 (Montreux (CH), 1993) (1993)

18. P.P. Mitra, J.B. Stark: Nonlinear limits to the information capacity of optical fibre communications. Nature 411(6841), 1027 (2001). DOI $10.1038 / 35082518$

19. A. Carena, G. Bosco, V. Curri, P. Poggiolini, M.T. Taiba, F. Forghieri: Statistical characterization of PM-QPSK signals after propagation in uncompensated fiber links. in 36th European Conference and Exhibition on Optical Communication, 1, p. P4.07 (IEEE, Torino (IT), 2010) (2010). DOI 10.1109/ECOC.2010.5621509

20. F. Vacondio, O. Rival, C. Simonneau, E. Grellier, A. Bononi, L. Lorcy, J.C. Antona, S. Bigo: On nonlinear distortions of highly dispersive optical coherent systems. Optics Express 20(2), 1022 (2012). DOI 10.1364/OE.20.001022

21. R. Dar, M. Feder, A. Mecozzi, M. Shtaif: Properties of nonlinear noise in long, dispersion-uncompensated fiber links. Optics Express 21(22), 25685 (2013). DOI 10.1364/OE.21.025685

22. R. Dar, M. Feder, A. Mecozzi, M. Shtaif: Accumulation of nonlinear interference noise in fiber-optic systems. Optics Express 22(12), 14199 (2014). DOI 10.1364/OE.22.014199

23. A. Carena, G. Bosco, V. Curri, Y. Jiang, P. Poggiolini, F. Forghieri: EGN model of non-linear fiber propagation. Optics Express 22(13), 16335 (2014). DOI 10.1364/OE.22.016335

24. H. Kogelnik, A. Yariv: Considerations of noise and schemes for its reduction in laser amplifiers. Proceedings of the IEEE 52(2), 165 (1964). DOI 10.1109/PROC.1964.2805

25. A. Yariv: Signal-to-noise considerations in fiber links with periodic or distributed optical amplification. Optics Letters 15(19), 1064 (1990). DOI 10.1364/OL.15.001064

26. P. Poggiolini, G. Bosco, A. Carena, V. Curri, Y. Jiang, F. Forghieri: The GN-Model of Fiber Non-Linear Propagation and its Applications. Journal of Lightwave Technology 32(4), 694 (2014). DOI 10.1109/JLT.2013.2295208

27. D.J. Ives, P. Bayvel, S.J. Savory: Adapting Transmitter Power and Modulation Format to Improve Optical Network Performance Utilizing the Gaussian Noise Model of Nonlinear Impairments. Journal of Lightwave Technology 32(21), 3485 (2014). DOI 10.1109/JLT.2014.2346582

28. P. Poggiolini: The GN Model of Non-Linear Propagation in Uncompensated Coherent Optical Systems. Journal 
of Lightwave Technology 30(24), 3857 (2012). DOI 10.1109/JLT.2012.2217729

29. A. Carena, G. Bosco: Impact of the transmitted signal initial dispersion transient on the accuracy of the GN-model of non-linear propagation. in European Conference and Exposition on Optical Communications, 1, p. Th.1.D.4 (London (UK), 2013) (2013)

30. E. Ip, J.M. Kahn: Compensation of Dispersion and Nonlinear Impairments Using Digital Backpropagation. Journal of Lightwave Technology 26(20), 3416 (2008). DOI 10.1109/JLT.2008.927791

31. ETSI. Network Aspects (NA); Availability performance of path elements of international digital paths (1998)

32. J.P. Snyder, Map Projections - A Working Manual. Tech. rep., U.S. Geological Survey Proffesional Paper 1395, Washington, D.C. (1987)

33. R. Ramaswami, K. Sivarajan: Design of logical topologies for wavelength-routed all-optical networks. in Proceedings of $I N$ FOCOM'95, p. 10c.4.1 (IEEE Comput. Soc. Press, Boston, MA (USA), 1995) (1995). DOI 10.1109/INFCOM.1995.516012

34. S. Jain, A. Kumar, S. Mandal, J. Ong, L. Poutievski, A. Singh, S. Venkata, J. Wanderer, J. Zhou, M. Zhu, J. Zolla, U. Hölzle, S. Stuart, A. Vahdat: B4 : Experience with a Globally-Deployed Software Defined WAN. in SIGCOMM (2013) (2013)

35. D. Monoyios, K. Vlachos: Multiobjective Genetic Algorithms for Solving the Impairment-Aware Routing and Wavelength Assignment Problem. Journal of Optical Communications and Networking 3(1), 40 (2011). DOI 10.1364/JOCN.3.000040

36. K. Cho, D. Yoon: On the general BER expression of oneand two-dimensional amplitude modulations. IEEE Transactions on Communications 50(7), 1074 (2002). DOI 10.1109/TCOMM.2002.800818

37. P.K. Vitthaladevuni, M.S. Alouini, J. Kieffer: Exact BER computation for cross QAM constellations. IEEE Transactions on Wireless Communications 4(6), 3039 (2005). DOI 10.1109/TWC.2005.857997

38. P. Poggiolini, G. Bosco, A. Carena, R. Cigliutti, V. Curri, F. Forghieri, R. Pastorelli, S. Piciaccia: The LOGON Strategy for Low-Complexity Control Plane Implementation in NewGeneration Flexible Networks. in Optical Fiber Communication Conference, 1, p. OW1H.3 (Anaheim, CA (USA), 2013) (2013). DOI 10.1364/OFC.2013.OW1H.3

39. D. Banerjee, B. Mukherjee: A practical approach for routing and wavelength assignment in large wavelength-routed optical networks. IEEE Journal on Selected Areas in Communications 14(5), 903 (1996). DOI 10.1109/49.510913

40. N. Wauters, P. Demeester: Design of the optical path layer in multiwavelength cross-connected networks. IEEE Journal on Selected Areas in Communications 14(5), 881 (1996). DOI 10.1109/49.510911

41. J.Y. Yen: Finding the K Shortest Loopless Paths in a Network. Management Science 17(11), 712 (1971). DOI 10.2307/2629312

42. P. Wright, A. Lord, S. Nicholas: Comparison of Optical Spectrum Utilization Between Flexgrid and Fixed Grid on a Real Network Topology. in Optical Fiber Communication Conference, p. OTh3B.5 (Los Angeles, CA. (USA), 2012) (2012). DOI 10.1364/OFC.2012.OTh3B.5 